\title{
ASYMPTOTICS OF COUNTS OF SMALL COMPONENTS IN RANDOM STRUCTURES AND MODELS OF COAGULATION-FRAGMENTATION
}

\author{
Boris L. GRAnOvskY ${ }^{1}$
}

\begin{abstract}
We establish necessary and sufficient conditions for the convergence (in the sense of finite dimensional distributions) of multiplicative measures on the set of partitions. The multiplicative measures depict distributions of component spectra of random structures and also the equilibria of classic models of statistical mechanics and stochastic processes of coagulation-fragmentation. We show that the convergence of multiplicative measures is equivalent to the asymptotic independence of counts of components of fixed sizes in random structures. We then apply Schur's tauberian lemma and some results from additive number theory and enumerative combinatorics in order to derive plausible sufficient conditions of convergence. Our results demonstrate that the common belief, that counts of components of fixed sizes in random structures become independent as the number of particles goes to infinity, is not true in general.
\end{abstract}

Mathematics Subject Classification. 60C05, 60K35, 05A16, 82B05, $11 \mathrm{M} 45$.

Received November 4, 2010. Revised September 18, 2011.

\section{Introduction: PRobabilistic SETting AND ITS APPLiCATIONS}

We start from the following formalism.

Let $\left\{Z_{j}, j \geq 1\right\}$ be a sequence of independent integer valued random variables that induces a sequence of random vectors $\left\{\mathbf{K}^{(n)}=\left(K_{1}^{(n)}, \ldots, K_{n}^{(n)}\right), n \geq 1\right\}$ given by

$$
\mathcal{L}\left(\mathbf{K}^{(n)}\right)=\mathcal{L}\left(Z_{1}, \ldots, Z_{n} \mid \sum_{j=1}^{n} j Z_{j}=n\right), \quad n=1,2, \ldots
$$

It follows from (1.1) that $\mathbf{K}^{(n)} \in \Omega_{n}, n \geq 1$, where

$$
\Omega_{n}=\left\{\eta=\left(k_{1}, \ldots, k_{n}\right): \sum_{j=1}^{n} j k_{j}=n\right\}
$$

is the set of all partitions $\eta$ of an integer $n$. In probabilistic combinatorics, (1.1) is called the conditioning relation (see [3]), while the sequence of vectors $\left\{\mathbf{K}^{(n)}, n \geq 1\right\}$, is called the counting process.

Keywords and phrases. Multiplicative measures on the set of partitions, random structures, coagulation-fragmentation processes, Schur's lemma, models of ideal gas.

1 Department of Mathematics, Technion-Israel Institute of Technology, 32000 Haifa, Israel. mar18aa@techunix.technion.ac.il 
Next, denote by $\mu_{n}$ the probability measure on $\Omega_{n}$ induced by the conditioning relation (1.1):

$$
\mu_{n}(\eta):=\mathbb{P}\left(\mathbf{K}^{(n)}=\eta\right), \quad \eta \in \Omega_{n}, \quad n \geq 1
$$

and let

$$
a_{k}^{(j)}=\mathbb{P}\left(Z_{j}=k\right), \quad k \geq 0, \quad j \geq 1 .
$$

We then have

$$
\mu_{n}(\eta)=c_{n}^{-1} \prod_{j=1}^{n} a_{k_{j}}^{(j)}, \quad \eta=\left(k_{1}, \ldots, k_{n}\right) \in \Omega_{n}, \quad n \geq 1,
$$

where

$$
c_{n}=\mathbb{P}\left(\sum_{j=1}^{n} j Z_{j}=n\right)=\sum_{\eta \in \Omega_{n}} \prod_{j=1}^{n} a_{k_{j}}^{(j)}, \quad \eta=\left(k_{1}, \ldots, k_{n}\right)
$$

is the partition function for the measure $\mu_{n}$. Taking into account (1.1), (1.4) we will assume throughout this paper that the probabilities $a_{k}^{(j)}, k \geq 0, j \geq 1$ are such that $c_{n}>0, n \geq 1$. Vershik [31] suggested that the class of measures (1.4) be called multiplicative, while Pitman [27] calls them Gibbsian (see also [21]). Observe that the multiplicative form (1.4) of the sequence of measures $\mu_{n}$ is implied by the fact that the random variables $Z_{j}, j \geq 1$ in (1.1) do not depend on $n$.

It is clear that the sequence of measures $\left\{\mu_{n}, n \geq 1\right\}$ induced by (1.1) is uniquely defined by the array of probabilities $\left\{a_{k}^{(j)}, j \geq 1, k \geq 0\right\}$. However, this correspondence is not a bijection. In fact, the "tilting" transformation (see [3]) of the probabilities:

$$
a_{k}^{(j)}(\rho)=\frac{\rho^{j k} a_{k}^{(j)}}{S^{(j)}(\rho)}, \quad k \geq 0, \quad j \geq 1
$$

where $\rho>0$ and $S^{(j)}(\rho)$ is the normalizing constant, does not change the sequence of measures $\left\{\mu_{n} n \geq 1\right\}$. But this transformation does affect the generic partition function $c_{n}$ leading to the tilted partition function $c_{n}(\rho)$ :

$$
c_{n}(\rho)=\frac{c_{n} \rho^{n}}{\prod_{j=1}^{n} S^{(j)}(\rho)}, \quad n \geq 1 .
$$

Note that the tilting is defined for all finite $\rho>0$ such that

$$
S^{(j)}(\rho)=\sum_{k=0}^{\infty} \rho^{j k} a_{k}^{(j)}<\infty, \quad j \geq 1
$$

It is a remarkable fact that the representation (1.1) provides a mathematical formalism for a variety of models in seemingly unrelated contexts. Let us briefly describe four main fields of application of this setting.

Decomposable combinatorial structures (for more details see $[3,19,26]$ and references therein). The size of such a structure is defined to be the number of elements in it. A decomposable structure of size $n$ is a union of indecomposable components (=components), so that the counts $k_{1}, \ldots, k_{n}$ of components of sizes $1, \ldots, n$ respectively, form an integer partition of $n$. It is assumed that each component of size $j$ belongs to one of $m_{j}$ types. The three classes of decomposable structures: assemblies, multisets and selections, encompass the whole universe of classical combinatorial objects. Assemblies are structures composed of labeled elements. The class of assemblies includes permutations decomposed into cycles $\left(m_{j}=(j-1) !\right)$, forests composed of rooted trees with labeled vertices $\left(m_{j}=j^{j-1}\right)$, graphs composed of connected subgraphs with labeled vertices $\left(m_{j} \sim 2^{\left(\begin{array}{l}j \\ 2\end{array}\right)}\right)$, etc. 
We note that for the last model $m_{j}$ appears to be asymptotically equal to the total number $2^{\left(\begin{array}{l}j \\ 2\end{array}\right)}$ of graphs on $j$ vertices. This follows from the remarkable fact that a random graph on $n$ vertices is connected with probability 1 , as $n \rightarrow \infty$ (see [9]). Multisets are formed from unlabeled elements. Examples of multisets are integer partitions $\left(m_{j}=1\right)$, planar partitions $\left(m_{j}=j\right)$ and mapping patterns $\left(m_{j} \sim \frac{\rho^{-j}}{2 j}, \rho=0.3383\right)$. Regarding the last example, recall that a mapping from the set $[1, n]$ to itself is a digraph with edges $(i, f(i), i=1, \ldots, n)$ decomposed into connected subgraphs of the underlying undirected graph. Mapping patterns are obtained from the above structure by removing labels, so that only the topology of the graph matters. Finally, selections are defined as multisets with distinct components, which means that all component counts $k_{j}, j=1, \ldots, n$ are either 0 or 1 . A typical example of a selection is an integer partition into distinct parts $\left(m_{j}=1\right)$.

The basic problem in enumerative combinatorics is to find the asymptotics, as $n$ goes to infinity, of the number of a certain class of structures of size $n$, with a component spectrum $\left(k_{1}, \ldots, k_{n}\right)$ in a given subset of $\Omega_{n}$. As a part of this problem, the asymptotics of the total number of given structures of size $n$ is of special interest.

The starting point of the probabilistic method considered is the definition of a random structure of size $n$, which is a random element $\Pi_{n}$ distributed uniformly on the finite set of all structures considered, with size $n$. Next is defined the induced random component spectrum $\mathbf{K}^{(n)}$, also called the counting process:

$$
\mathbf{K}^{(n)}=\mathbf{K}^{(n)}\left(\Pi_{n}\right)=\left(K_{1}^{(n)}, \ldots, K_{n}^{(n)}\right), n \geq 1, \quad \mathbf{K}^{(n)}\left(\Pi_{n}\right) \in \Omega_{n}
$$

where the random variable $K_{j}^{(n)}$ represents the number of components of size $j$ in $\Pi_{n}$.

It turns out that the representation (1.1) of the distribution of $\mathbf{K}^{(n)}$ is valid for the aforementioned three classes of combinatorial structures. Namely, assemblies, multisets and selections are induced respectively, by the following three types of random variables $Z_{j}, j \geq 1$ : Poisson $\left(\operatorname{Po}\left(a_{j}\right), a_{j}=\frac{m_{j}}{j !}\right)$, Negative Binomial $\left(N B\left(p^{j}, m_{j}\right)\right)$ and Binomial $\left(B i\left(\frac{p^{j}}{1+p^{j}}, m_{j}\right)\right), 0<p<1$.

Models of ideal gas (for references see [23,31] and [29], Chap. 12). In classical statistical mechanics, an ideal gas is a collection of perfectly elastic particles (atoms or molecules) which collide but otherwise do not interact with each other. It is assumed that the total internal energy $E$ of a gas is the sum of the microscopic energies of random motions of individual particles and that $E$ is partitioned between the particles, so that $k_{j}$, called an occupation number, is the number of particles with the energy level $j$, having a prescribed weight $m_{j}$ that regularly varies with $j$. The following three basic models (=statistics) of ideal gas are common.

Maxwell-Boltzmann (MB) (=labeled particles), Bose-Einstein (BE) (=indistinguishable particles), FermiDirac (FD) (=indistinguishable particles, such that no more than one particle may have a given energy level). In accordance with the setting for combinatorial structures, MB, BE and FD models conform to assemblies, multisets and selections, respectively. The probability distribution of the energy states $\eta$ which varies from model to model, is defined by a measure on the state space $\Omega_{n}$. By laws of statistical mechanics, these measures are forced to be of the multiplicative form (1.4), with the numbers $a_{k}^{(j)}$ defining the type of a model of the ideal gas considered.

A substantial difference of the model of ideal gas, treated as a quantum system is that a particle of the $d$-dimensional gas is viewed as a lattice point $\mathbf{q} \in \mathcal{Z}_{d}$ and the energy levels $\epsilon_{\mathbf{q}}$, called energy eigenvalues, are of the following special form:

$$
\epsilon_{\|\mathbf{q}\|^{2}}=c\|\mathbf{q}\|^{2}, \mathbf{q}=\left(l_{1}, \ldots, l_{d}\right) \in \mathcal{Z}^{d}, \quad\|\mathbf{q}\|^{2}=\sum_{s=1}^{d} l_{s}^{2},
$$

where $c>0$ is a known constant that does not depend on q. Consequently, the state of the quantum system is determined by a weighted partition $\eta=\left(k_{1}, \ldots, k_{n}\right)$ of an integer $n=E: E=\sum_{j \geq 1} j k_{j}$. By (1.8), to each 
energy level $j$ is naturally prescribed a "weight" $r_{d}(j)$ which is the number of representations of the natural number $j$ as the sum of $d$ integer squares. In other words, $r_{d}(j)$ is the number of distinct lattice positions $\mathbf{q}=\left(l_{1}, \ldots, l_{d}\right) \in \mathcal{Z}^{d}$ of a particle on a sphere of radius $\sqrt{j}$, i.e. with the energy level $j$. It is known from number theory (see e.g. [24]) that for $d \geq 5$,

$$
C_{1} j^{\frac{d-2}{2}} \leq r_{d}(j) \leq C_{2} j^{\frac{d-2}{2}}, \quad j \geq 1,
$$

where $C_{1}, C_{2}$ are positive constants depending on $d$, and that for $d=2,3,4$ the functions $r_{d}(j)$ oscillate wildly (in $j$ ), while, obviously,

$$
r_{1}(j)= \begin{cases}2, & \text { if } j \text { is a square } \\ 0, & \text { otherwise. }\end{cases}
$$

Employing known properties of $r_{d}(j)$, an important fact was proven in [32] that for the sake of asymptotic analysis, it is possible to treat the $d$ - dimensional quantum models as the classic $B E$ and $F D$ ones with parameters $m_{j}=c j^{\beta}$, where $c>0$, and $\beta=\frac{d-2}{2}$, if $d \geq 2$.

Coagulation-fragmentation processes on the set of integer partitions (see $[3,14]$ ). We will show that a multiplicative measure $\mu_{n}$ can be viewed as an equilibrium of a classic coagulation-fragmentation process (CFP) which is a time-continuous Markov chain on $\Omega_{n}$, defined as follows. A state $\eta=\left(k_{1}, \ldots, k_{n}\right) \in \Omega_{n}$ of a CFP depicts a partition of a total number $n$ of identical particles (animals, atoms, stars, human beings, etc) into clusters (=groups) of different sizes, so that $k_{j}$ is the number of clusters of size $j$. The only possible infinitesimal (in time) transitions are coagulation (merging) of two clusters of sizes $i$ and $j$ into one cluster of size $i+j$ and fragmentation (splitting) of a cluster of size $i+j$ into two clusters of sizes $i$ and $j$. Given a state $\eta \in \Omega_{n}$, with $k_{i}, k_{j}>0$ for some $1 \leq i, j \leq n$, denote by $\eta^{(i, j)} \in \Omega_{n}$ the state that is obtained from $\eta$ by the coagulation of some two clusters of sizes $i$ and $j$, and denote by $u_{c}\left(\eta, \eta^{(i, j)}\right)$ the rate of the infinitesimal transition $\eta \rightarrow \eta^{(i, j)}$. Similarly, for a given state $\eta \in \Omega_{n}$ with $k_{i+j}>0$, let $\eta_{(i, j)}$ be the state that is obtained from $\eta$ by the fragmentation of some cluster of size $i+j$ into two clusters of sizes $i$ and $j$, and let $u_{f}\left(\eta, \eta_{(i, j)}\right)$ be the rate of the infinitesimal transition $\eta \rightarrow \eta_{(i, j)}$. Denoting by

$$
q(\eta ; i, j)=\frac{u_{c}\left(\eta, \eta^{(i, j)}\right)}{u_{f}\left(\eta^{(i, j)}, \eta\right)}
$$

the ratio of the above transitions, the important property of reversibility of multiplicative measures is derived by verifying the detailed balance condition.

Proposition 1.1. A multiplicative measure $\mu_{n}$ defined by (1.4) is reversible with respect to the transition rates $u_{c}, u_{f}$ if their ratio satisfies:

$$
q(\eta ; i, j)= \begin{cases}\frac{a_{k_{i}-1}^{(i)} a_{k_{j}-1}^{(j)} a_{k_{i+j}}^{(i+j)}}{a_{k_{i}}^{(i)} a_{k_{j}}^{(j)} a_{k_{i+j}}^{(i+j)}}, & \text { if } i \neq j: k_{i}, k_{j}>0 \\ \frac{a_{k_{i}-2}^{(i)} a_{k_{2 i}+1}^{(2 i)}}{a_{k_{i}}^{(i)} a_{k_{2 i}}^{(2 i)}}, & \text { if } i=j: k_{i} \geq 2 .\end{cases}
$$

An immediate consequence of Proposition 1.1 is that a multiplicative measure $\mu_{n}$ defined by (1.4) is the equilibrium distribution of a CFP with transition rates $u_{c}, u_{f}$ obeying the condition (1.10), under some sequence of probabilities $\left\{a_{k_{j}}^{(j)}\right\}$. 
We now distinguish a class of CFP's with transition rates $u_{c}, u_{f}$ of the form

$$
\begin{aligned}
& u_{c}\left(\eta, \eta^{(i, j)}\right)=\left\{\begin{array}{lll}
k_{i} k_{j} \phi(i, j), & \text { if } i \neq j, & k_{i} k_{j}>0 \\
k_{i}\left(k_{i}-1\right) \phi(i, i), & \text { if } i=j, & k_{i} \geq 2,
\end{array}\right. \\
& u_{f}\left(\eta, \eta_{(i, j)}\right)=k_{i+j} \varphi(i, j), \quad 2 \leq i+j \leq n, \quad k_{i+j} \geq 2,
\end{aligned}
$$

where $\phi, \varphi$ are some symmetric nonnegative functions on the set of pairs of positive integers. Treating the functions $\phi, \varphi$ in (1.11) as the rates of a single coagulation and a single fragmentation respectively, the induced CFP's can be viewed as mean-field models on the set $\Omega_{n}$. In fact, (1.11) tells us that at any state $\eta \in \Omega_{n}$, each cluster may coagulate with every other one or may be fragmented into two parts, so that the net rates of the transitions $\eta \rightarrow \eta_{(i, j)}$ and $\eta \rightarrow \eta^{(i, j)}$ are sums of the rates of all possible single coagulations and fragmentations respectively, at the state $\eta$. It is proven in [14] with the help of the Kolmogorov cycle condition that in the case when all rates of single coagulations and fragmentations are positive, the CFP's given by (1.11) are reversible if and only if the ratios of the single transitions are of the form

$$
\frac{\phi(i, j)}{\varphi(i, j)}=\frac{a_{i+j}}{a_{i} a_{j}}, \quad i, j \geq 1
$$

with some $a_{i}>0, \quad i \geq 1$. The corresponding CFP's are known as classical reversible models of clustering and networks studied in 1970-s by Kelly and Whittle (see [14-16] and references therein). The equilibrium measures of the mean-field CFP's with the rates (1.11), (1.12) are multiplicative measures $\mu_{n}$ induced by the conditioning relation (1.1) with $Z_{j}$ distributed $P_{o}\left(a_{j}\right), j \geq 1$. An example of a reversible CFP which is not a mean field, is provided by setting in (1.3) $Z_{j}=G_{j}-1$, where $G_{j}$ is distributed geometrically with parameter $p^{j}, 0<p<1$. Then $a_{k}^{(j)}=p^{j k} q_{j}, q_{j}=1-p^{j}, j \geq 1, k \geq 0$, and the corresponding measure $\mu_{n}$ is the uniform one on the set $\Omega_{n}$, while in (1.10), $q(\eta ; i, j) \equiv 1$. It is simple to see that, due to the last fact, the net transition rates of form (1.11) do not provide the detailed balance condition for the CFP considered in the example, which implies that the above reversible CFP is not a mean -field model.

CFP's on set partitions $([7,8,27])$. We assume here that in the preceding set up for CFP's, particles are labeled by $1, \ldots, n$, so that the state space of the system of clusters related to a CFP, becomes the set $\Omega_{[n]}=\left\{\pi_{[n]}\right\}$ of all partitions $\pi_{[n]}$ of the set $[n]=\{1, \ldots, n\}$ into subsets. Recall that a partition of $[n]$ into $k$ blocks (clusters) $A_{1}, \ldots, A_{k}$ is $\pi_{[n], k}=\left(A_{1}, \ldots, A_{k}\right)$, where $A_{j}, 1 \leq j \leq k \leq n$ are nonempty and disjoint subsets of $[n]$ whose union is $[n]$ and which are numbered, e.g. in the order of their least element. Denoting $\left|A_{j}\right|$ the size of a cluster $A_{j}$, we further assign to each $A_{j}$, a weight $m_{\left|A_{j}\right|}$ which is a number of possible states of $A_{j}$, the states can be e.g., shapes (in the plane or in space), colors, energy levels, etc. This says that to the set partition $\pi_{[n], k}$ correspond $\prod_{j=1}^{k} m_{\left|A_{j}\right|}$ different structures with the same blocks $A_{1}, \ldots, A_{k}$, so that the total number of structures formed by all partitions of the set $[n]$ into $k$ given clusters is equal to

$$
\sum_{\pi_{[n], k} \in \Omega_{[n], k}} \prod_{j=1}^{k} m_{\left|A_{j}\right|}:=B_{n, k},
$$

where $B_{n, k}$ is known as a Bell polynomial in weights $m_{1}, \ldots, m_{n-k+1}$. Similar to the setting for decomposable combinatorial structures, a random structure $\Pi_{[n], k}$ is the one chosen randomly from the set of $B_{n, k}$ structures. As a result, for a given $k$ a measure $p_{[n], k}$ on the set $\Omega_{[n], k}=\left\{\pi_{[n], k}\right\}$ is induced:

$$
p_{[n], k}\left(\pi_{[n]}\right)=\frac{\prod_{j=1}^{k} m_{\left|A_{j}\right|}}{B_{n, k}}, \quad \pi_{[n]} \in \Omega_{[n], k} .
$$

In a more general setting which encompasses a variety of models (see $[7,27]$ ), the weights $m_{j}$ in $(1.14)$ are allowed to be arbitrary nonnegative numbers. Pitman [27] calls the $\Pi_{[n], k}$ a Gibbs partition and the measure 
$p_{[n], k}$ microcanonical Gibbs distribution. Obviously, the vector $\left(\left|A_{1}\right|, \ldots\left|A_{k}\right|\right)$ of block size counts defines a partition of the integer $n$ into $k$ summands, induced by the generic set partition $\pi_{[n], k}$, and it is known that to each $\eta=\left(k_{1}, \ldots, k_{n}\right) \in \Omega_{n}$, such that $k_{1}+\ldots+k_{n}=k$, correspond

$$
\frac{n !}{\prod_{j=1}^{n}\left(k_{j} !\right)(j !)^{k_{j}}}
$$

different set partitions $\pi_{[n], k} \in \Omega_{[n], k}$, each one of them having the same probability $p_{[n], k}\left(\pi_{[n], k}\right)$ given by $(1.14)$. Thus, the Gibbs distribution $p_{[n], k}$ on $\Omega_{[n], k}$ induces the Gibbs distribution $p_{n, k}$ on the set $\Omega_{n, k}$ of integer partitions of $n$ into $k$ positive summands:

$$
p_{n, k}(\eta)=\left(B_{n, k}\right)^{-1} \prod_{j=1}^{n}\left(\frac{m_{j}}{j !}\right)^{k_{j}} \frac{1}{\left(k_{j}\right) !}, \quad \eta=\left(k_{1}, \ldots, k_{n}\right) \in \Omega_{n, k},
$$

where the partition function $B_{n, k}$ defined as in (1.13) can be rewritten in the following form:

$$
B_{n, k}=\sum_{\eta \in \Omega_{n, k}} \prod_{j=1}^{n}\left(\frac{m_{j}}{j !}\right)^{k_{j}} \frac{1}{\left(k_{j}\right) !}
$$

From (1.15) it is easy to derive Kolchin's representation of Gibbs partitions (see [27], Thm. 1.2). On the other hand, the distribution $p_{n, k}$ given by (1.15) is produced by conditioning the multiplicative measure $\mu_{n}$ defined by (1.4) on the event $Z_{1}+\ldots+Z_{n}=k$, with $Z_{j} \sim \operatorname{Po}\left(a_{j}\right), a_{j}=\frac{m_{j}}{j !}, j \geq 1$. However, this embedding of the generic model associated with set partitions of $[n]$ into the setting for conditioning relation (1.1) does not facilitate the study of a wealth of problems (see [7]) arising from treating $p_{[n], k}, k=1, \ldots, n$ as marginal distributions of irreversible time continuous markov processes of pure fragmentation (or pure coagulation) on the state space $[n]$. The study of these problems was initiated by Kingman and Pitman and has been extensively continued by a group of researchers including Pitman, Bertoin, Berestycki, Gnedin et al.

In what follows we will refer to all models induced by the conditioning relation (1.1) as random structures (RS's).

\section{OBJECTIVE AND SUMMARY}

In this paper, we study the asymptotic behaviour, as $n \rightarrow \infty$, of the random vector $\left(K_{1}^{(n)}, \ldots, K_{l}^{(n)}\right)$ composed of the first $l \geq 1$ components of the random vector $\mathbf{K}^{(n)}$, defined by (1.1). In view of the independence of the random variables $Z_{j}, j \geq 1$ in (1.1), there was a common belief in physics and combinatorics that the small (compared with $n$ ) counts $K_{1}^{(n)}, \ldots, K_{l}^{(n)}$ become independent, as $n \rightarrow \infty$, for any fixed $l \geq 2$, and this was proven in a variety of particular cases of RS's. We show that in general the assumption of asymptotic independence fails. This said, we note that properly scaled large component counts $K_{l}^{(n)}, K_{l+1}^{(n)}, \ldots, K_{n}^{(n)}$ are known to be dependent in the limit, for any fixed $l \geq 1$. Our main result which is Theorem 3.3 in Section 3, consists of establishing the necessary and sufficient conditions for the asymptotic independence of small component counts. Combining this result with the Schur's lemma we provide in Section 4 a plausible sufficient condition for convergence RS's. This allows us to answer the question of convergence of counting processes for the three basic types of RS's discussed in Section 1. It turns out that many models of RS's are divergent. In a parallel way we discuss the problem of convergence for CFP's. The final section, Section 5, contains concluding remarks, among them a historical background of the problem. 


\section{MAin RESUlT}

Definition 3.1. We say that the counting process $\left\{\mathbf{K}^{(n)}, n \geq 1\right\}$, is convergent, if for each fixed $l \geq 1$, the probability law $\mathcal{L}\left(K_{1}^{(n)}, \ldots, K_{l}^{(n)}\right)$ weakly converges, as $n \rightarrow \infty$, to some probability law $F_{l}$ on $\mathbb{R}^{l}, l \geq 1$. Moreover, we say that the counts $K_{1}^{(n)}, \ldots, K_{l}^{(n)}, l \geq 2$ of small components of the random vector $\mathbf{K}^{(n)}$ are asymptotically independent if the above laws $F_{l}$ are product measures on $\mathbb{R}^{l}$, for all finite $l \geq 2$.

Note that in contrast to the setting for limit shapes (see e.g. $[17,31]$ ), in this paper we are interested in the weak convergence of non scaled multiplicative measures.

For a fixed $l \geq 1$, given $k_{1}, \ldots, k_{l}$ and sufficiently large $n$, we denote

$$
M_{l}=\sum_{j=1}^{l} j k_{j}, \quad \text { and } \quad T^{(l)}\left(n, M_{l}\right)=\mathbb{P}\left(\sum_{j=l+1}^{n-M_{l}} j Z_{j}=n-M_{l}\right) .
$$

It is immediate that

$$
T^{(l)}\left(n, M_{l}\right)=T^{(l)}\left(n-M_{l}, 0\right):=T_{n-M_{l}}^{(l)}, \quad l \geq 1, \quad M_{l} \geq 0 .
$$

Assuming in what follows that

$$
a_{0}^{(j)}>0, j \geq 1
$$

we will be dealing with the "scaled" quantities $\tilde{a}_{k}^{(j)}, \tilde{c}_{n}$, and $\tilde{T}_{n-k}^{(l)}$ defined by

$$
\begin{gathered}
\tilde{a}_{k}^{(j)}=\frac{a_{k}^{(j)}}{a_{0}^{(j)}}, \quad k \geq 0, \quad j \geq 1, \\
\tilde{c}_{n}=\left(\prod_{j=1}^{n} a_{0}^{(j)}\right)^{-1} c_{n}, \quad n \geq 1, \quad \tilde{c}_{0}=1, \\
\tilde{T}_{n-k}^{(l)}=\left(\prod_{j=l+1}^{n-k} a_{0}^{(j)}\right)^{-1} T_{n-k}^{(l)}, \quad \tilde{T}_{0}^{(l)}=1, \quad l \geq 1, \quad 0 \leq k \leq n .
\end{gathered}
$$

In the context of decomposable combinatorial structures, the quantities $\tilde{c}_{n}$ and $\tilde{T}_{n-k}^{(l)}$ have a significant combinatorial meaning. Denoting by $p_{n}$ the number of structures of size $n$, we demonstrate in Section 4 that

$$
p_{n}= \begin{cases}p^{-n} \tilde{c}_{n}, & \text { for multisets }\left(N B\left(p^{j}, m_{j}\right)\right) \text { and selections }\left(B i\left(p^{j}, m_{j}\right)\right) \\ n ! \tilde{c}_{n}, & \text { for assemblies }\left(P o\left(a_{j}\right)\right) .\end{cases}
$$

In analogous way, $\tilde{T}_{n-k}^{(l)}$ is related to the number of structures of size $(n-k)$ with all component sizes greater than $l$.

With the help of the above notation, we have

$$
\mathbb{P}\left(K_{1}^{(n)}=k_{1}, \ldots, K_{l}^{(n)}=k_{l}\right)=c_{n}^{-1}\left(\prod_{j=1}^{l} a_{k_{j}}^{(j)}\right) \mathbb{P}\left(\sum_{j=l+1}^{n} j Z_{j}=n-M_{l}\right)=\left(\prod_{j=1}^{l} \tilde{a}_{k_{j}}^{(j)}\right) \frac{\tilde{T}_{n-M_{l}}^{(l)}}{\tilde{c}_{n}}
$$

where in the last step we have used the fact that

$$
\mathbb{P}\left(\sum_{j=l+1}^{n} j Z_{j}=n-M_{l}\right)=T_{n-M_{l}}^{(l)} \prod_{k=1}^{M_{l}} \mathbb{P}\left(Z_{n-M_{l}+k}=0\right)
$$


and the definitions (3.3), (3.4) and (3.5) of the "scaled" quantities. Note that in view of $(3.1), \tilde{T}_{n-M_{l}}^{(l)}$ is the same for all $k_{1}, \ldots, k_{l}: \sum_{j=1}^{l} j k_{j}=M_{l}$.

Central to our subsequent study is the notion of smoothly growing real sequences $R T_{\rho}$, the definition of which we adopt from $[6,10]$.

Definition 3.2. $R T_{\rho}, 0 \leq \rho \leq \infty$ is the collection of sequences $\left\{d_{n}\right\}_{n \geq 1}$ of nonnegative numbers that satisfy

$$
\lim _{n \rightarrow \infty} \frac{d_{n}}{d_{n+1}}=\rho
$$

Sequences in $R T_{\rho}$ play a key role in Compton's theory of logical limit laws and in additive number theory (for references see $[5,6,10])$.

Now we are prepared to state our main result.

Theorem 3.3. The counting process $\left\{\mathbf{K}^{(n)}, n \geq 1\right\}$ is convergent if and only if the following two conditions hold:

(a) $\left\{\tilde{c}_{n}\right\}_{n \geq 0} \in R T_{\rho}$, for some $0 \leq \rho<\infty$; and

(b) For each $l \geq 1$ there exists a positive finite limit

$$
q^{(l)}=\lim _{n \rightarrow \infty} \frac{\tilde{T}_{n}^{(l)}}{\tilde{c}_{n}} .
$$

Moreover, counts of small components of a convergent random vector $\mathbf{K}^{(n)}$ are asymptotically independent.

Proof. In view of (3.6), the counting process $\left\{\mathbf{K}^{(n)}, n \geq 1\right\}$ is convergent if and only if the fraction on the RHS of (3.6) has finite limits, as $n \rightarrow \infty$ for all fixed $M_{l} \geq 0, l \geq 1$, while for any $l \geq 1$ there exists an $M_{l} \geq 0$, such that

$$
\lim _{n \rightarrow \infty} \frac{\tilde{T}_{n-M_{l}}^{(l)}}{\tilde{c}_{n}}>0 .
$$

We note that (3.9) secures that the limiting distribution is a probability measure. Next we write

$$
\frac{\tilde{T}_{n-M_{l}-1}^{(l)}}{\tilde{c}_{n}}=\left(\frac{\tilde{T}_{n-M_{l}-1}^{(l)}}{\tilde{c}_{n-1}}\right)\left(\frac{\tilde{c}_{n-1}}{\tilde{c}_{n}}\right) .
$$

We first prove the necessity of the conditions $(a),(b)$ of the theorem. Assuming that $\left\{\mathbf{K}^{(n)}, n \geq 1\right\}$ converges, it follows from (3.6) that there exists a finite limit

$$
\lim _{n \rightarrow \infty} \mathbb{P}\left(K_{1}^{(n)}=0, \ldots, K_{l}^{(n)}=0\right)=\lim _{n \rightarrow \infty} \frac{\tilde{T}_{n}^{(l)}}{\tilde{c}_{n}}:=q^{(l)}<\infty, \quad l \geq 1 .
$$

Consequently, (3.10), (3.9) and the preceding discussion imply that $\tilde{c}_{n} \in R T_{\rho}$, for some $0 \leq \rho<\infty$ and we get from (3.10)

$$
\lim _{n \rightarrow \infty} \frac{\tilde{T}_{n-M_{l}}^{(l)}}{\tilde{c}_{n}}=q^{(l)} \rho^{M_{l}}, l \geq 1
$$

for all $0 \leq M_{l}<\infty$. From the latter and (3.9) we conclude that $q^{(l)}$ is positive. For the proof of sufficiency we first apply (3.10) with $M_{l}=0$ to conclude, by virtue of the conditions (a) and (b), that (3.11) holds with $M_{l}=1, l \geq 1$ and so on, proving (3.11) for all $M_{l} \geq 0$. As a result,

$$
\lim _{n \rightarrow \infty} \mathbb{P}\left(K_{1}^{(n)}=k_{1}, \ldots, K_{l}^{(n)}=k_{l}\right)=q^{(l)} \prod_{j=1}^{l} \tilde{a}_{k_{j}}^{(j)} \rho^{j k_{j}}, \quad l \geq 1,
$$


by (3.6) and the definition of $M_{l}$. Since the sum over $\left(k_{1}, \ldots, k_{l}\right) \in \mathbb{R}^{l}$ of the LHS of (3.12) is equal to 1 , we obtain the explicit expression for $q^{(l)}$ :

$$
q^{(l)}=\left(\prod_{j=1}^{l} \tilde{S}^{(j)}(\rho)\right)^{-1}, \quad l \geq 1,
$$

where we denoted $\tilde{S}^{(j)}(\rho)=\sum_{k \geq 0} \tilde{a}_{k_{j}}^{(j)} \rho^{j k_{j}}$, in accordance with (1.7). This shows that the limiting distribution of the probability law $\mathcal{L}\left(K_{1}^{(n)}, \ldots, K_{l}^{(n)}\right)$ is the product probability measure

$$
\prod_{j=1}^{l} \frac{\tilde{a}_{k_{j}}^{(j)} \rho^{j k_{j}}}{\tilde{S}^{(j)}(\rho)}, \quad l \geq 1
$$

\section{Remark 3.4.}

(i) Setting $0^{0}=1$ and recalling that $\tilde{a}_{0}^{(j)}=1, j \geq 1$, it follows from (3.6) and (3.14) that in the case of a convergent counting process with $\rho=0$ in condition (a), the limit law of the random probability vector $\left(K_{1}^{(n)}, \ldots, K_{l}^{(n)}\right), l \geq 1$ is the measure concentrated at the singleton $(0, \ldots, 0) \in \mathbb{R}^{l}$. We also observe that in this case $q^{(l)}=1, l \geq 1$, in accordance with (3.12), while $\lim _{n \rightarrow \infty} \frac{\tilde{T}_{n-M_{l}}^{(l)}}{\tilde{c}_{n}}=0, l \geq 1$, for all $M_{l}>0$;

(ii) condition (c) implies $\left\{\tilde{T}_{n}^{(l)}\right\}_{n \geq 0} \in R T_{\rho}$, for all $l \geq 1$, with the same $0 \leq \rho<\infty$ as for the sequence $\left\{\tilde{c}_{n}\right\}_{n \geq 0}$. This can be seen by writing

$$
\frac{\tilde{T}_{n}^{(l)}}{\tilde{c}_{n}}=\left(\frac{\tilde{T}_{n}^{(l)}}{\tilde{T}_{n-1}^{(l)}}\right)\left(\frac{\tilde{T}_{n-1}^{(l)}}{\tilde{c}_{n-1}}\right)\left(\frac{\tilde{c}_{n-1}}{\tilde{c}_{n}}\right)
$$

and then applying the fact that $0<q^{(l)}<\infty$. Conversely, if (3.8) holds and $\left\{\tilde{T}_{n}^{(l)}\right\}_{n \geq 0} \in R T_{\rho}$, for some $l \geq 1$ and $0 \leq \rho<\infty$, then $\left\{\tilde{c}_{n}\right\}_{n \geq 0} \in R T_{\rho}$, with the same $\rho$;

(iii) one can see from the proof of Theorem 3.3 that the condition $q^{(l)}>0, l \geq 1$ which is a part of the condition (c), ensures the tightness of the corresponding sequences of finite dimensional probability measures.

To formulate the forthcoming corollary, we need to extend the definition (1.5) of the tilting transformation to the case $\rho=0$, in the following natural way:

$$
a_{k}^{(j)}(0)= \begin{cases}1, & \text { if } k=0 \\ 0, & \text { otherwise. }\end{cases}
$$

Corollary 3.5. Let $\mathbf{K}^{(n)}$ be a convergent counting process, such that $\tilde{c}_{n} \in R T_{\rho}$, for some $0 \leq \rho<\infty$. Then

$$
\lim _{n \rightarrow \infty} \mathbb{P}\left(K_{1}^{(n)}=k_{1}, \ldots, K_{l}^{(n)}=k_{l}\right)=\prod_{j=1}^{l} a_{k_{j}}^{(j)}(\rho), \quad l \geq 1,
$$

where $a_{k_{j}}^{(j)}(\rho)$ are the generic probabilities $a_{k_{j}}^{(j)}$ tilted with the above $\rho$.

Proof. By (3.8) and the definitions (1.5), (3.15) and (3.3), it follows from Theorem 3.3 that for a convergent counting process,

$$
\lim _{n \rightarrow \infty} \mathbb{P}\left(K_{1}^{(n)}=k_{1}, \ldots, K_{l}^{(n)}=k_{l}\right)=\prod_{j=1}^{l} \frac{\tilde{a}_{k_{j}}^{(j)} \rho^{j k}}{\tilde{S}^{(j)}(\rho)}=\prod_{j=1}^{l} \frac{a_{k_{j}}^{(j)} \rho^{j k}}{S^{(j)}(\rho)}=\prod_{j=1}^{l} a_{k_{j}}^{(j)}(\rho), \quad l \geq 1 .
$$




\section{Remark 3.6.}

(i) Corollary 3.5 says that asymptotic independence with the limit product measure composed of generic probabilities $a_{k_{j}}^{(j)}$ takes place only if $\left\{\tilde{c}_{n}\right\} \in R T_{1}$.

(ii) We denote by $c_{n}(\theta)$ the quantity $c_{n}$ corresponding to the tilting of the probabilities $a_{k}^{(j)}$ with $\theta \geq 0$ and recall that a multiplicative measure $\mu_{n}$ is invariant under all possible tiltings of the probabilities with $\theta>0$. By definitions (1.5) and (3.4) we then have

$$
\widetilde{c_{n}(\theta)}=\theta^{n} \tilde{c}_{n}, n \geq 0,
$$

where $\widetilde{c_{n}(\theta)}$ is the scaling of $c_{n}(\theta)$. So, if $\left\{\tilde{c}_{n}\right\}_{n \geq 0}, \in R T_{\rho}$, for some $0 \leq \rho<\infty$, then

$$
\left\{c_{n}(\theta)\right\}_{n \geq 0} \in R T_{\frac{\rho}{\theta}} \text {. }
$$

This clarifies the following meaning of Corollary 3.5. Consider a convergent counting process such that $\left\{\tilde{c}_{n}\right\}_{n \geq 0} \in R T_{\rho}$, with some $\rho>0$. Then the whole family of counting processes obtained by tilting the original one with all possible $\theta>0$, has the same limit finite dimensional distributions as the counting process obtained by tilting the original one with the $\rho>0$, so that the corresponding quantity $\left\{\widetilde{c_{n}(\rho)}, n \geq 0\right\} \in R T_{1}$.

\section{Convergent And Divergent Random structures}

We agree to call a RS convergent/divergent if the corresponding counting process converges/diverges in the sense of Definition 3.1.

Assuming that condition (a) of Theorem 3.3 holds, our tool for verifying condition (b) for the models considered will be the remarkable Schur tauberian lemma cited below. With an obvious abuse of notation, we say that a power series $f(x)=\sum_{n \geq 0} d_{n} x^{n}$ is in $R T_{\rho}$ if $\left\{d_{n}\right\}_{n \geq 0} \in R T_{\rho}$. We denote by * the Cauchy product, which is extended to formal power series as usual (see [10]).

Lemma 4.1 (Schur, see [10], p. 62). Let $f=f_{1} * f_{2}$, where $f, f_{1}, f_{2}$ are power series with coefficients $d_{n}, d_{n}^{(1)}, d_{n}^{(2)}, n \geq 0$, respectively,

such that:

(a) $f_{1} \in R T_{\rho}$ for some $0 \leq \rho<\infty$; and

(b) the radius of convergence of $f_{2}$ is greater than $\rho$.

Then

$$
\lim _{n \rightarrow \infty} \frac{d_{n}}{d_{n}^{(1)}}=f_{2}(\rho)
$$

Schur's lemma is widely used in asymptotic enumeration and in the study of asymptotic densities of additive number systems (see [10]). The proof of the lemma is quite simple (see [28], problem 178). In [35] a version of Schur's lemma for Dirichlet series was obtained, which allowed applications to multiplicative number theory.

We note the fact that, under the conditions of Schur's lemma, $f_{1} \in R T_{\rho}$ implies $f \in R T_{\rho}$. This can be seen by writing

$$
\frac{d_{n-1}}{d_{n}}=\left(\frac{d_{n-1}^{(1)}}{d_{n}^{(1)}}\right)\left(\frac{d_{n-1}}{d_{n-1}^{(1)}}\right)\left(\frac{d_{n}^{(1)}}{d_{n}}\right) .
$$

We now outline the scheme of application of Schur's lemma to our specific setting. Treating $S^{(j)}$ in $(1.7)$ as the generating probability function of the random variable $j Z_{j}$ (=of the sequence of probabilities $\left\{a_{k}^{(j)}\right\}$ ) in the conditioning relation (1.1), we have:

$$
S^{(j)}(x)=\sum_{k \geq 0} a_{k}^{(j)} x^{k j}
$$


Clearly, the radius of convergence of $S^{(j)}$ is $\geq 1$, for all $j \geq 1$. Next, we denote $\tilde{S}^{(j)}=\frac{1}{a_{0}^{(j)}} S^{(j)}, \tilde{g}=\prod_{j \geq 1} \tilde{S}^{(j)}$ and $\tilde{g}_{\tilde{T}^{(l)}}=\prod_{j \geq l+1} \tilde{S}^{(j)}$. Since $\tilde{a}_{0}^{(j)}=1, j \geq 1$, the above are the generating functions for the scaled sequences $\left\{\tilde{a}_{k}^{(j)}\right\}_{k \geq 0},\left\{\tilde{c}_{n}\right\}_{n \geq 0}$ and $\left\{\tilde{T}_{n}^{(l)}\right\}_{n \geq 0}$ respectively, as defined by (3.3)-(3.5). Finally, writing $\tilde{g}^{(l)}=\prod_{j=1}^{l} \tilde{S}^{(j)}, l \geq 1$ we have

$$
\tilde{g}=\tilde{g}_{\tilde{T}^{(l)}} * \tilde{g}^{(l)}
$$

or, equivalently,

$$
\tilde{g}_{\tilde{T}^{(l)}}=\tilde{g} *\left(\frac{1}{\tilde{g}^{(l)}}\right)
$$

Remark 4.2. We make here use of the representation (4.2) to show that condition (a) of Theorem 3.3 does not imply even the existence of the limit (3.8) defining $q^{(l)}, l \geq 1$. Let $l=1$,

$$
\tilde{g}(x)=\frac{1}{1-x}, \quad \tilde{g}^{(1)}(x)=1+x, \quad \tilde{g}_{\tilde{T}^{(1)}}(x)=\frac{1}{1-x^{2}}, \quad|x|<1 .
$$

Then

$$
\tilde{c}_{n}=1, \quad n \geq 1, \quad \tilde{T}_{n}^{(1)}= \begin{cases}0, & \text { if } n \text { is odd } \\ 1, & \text { if } n \text { is even } n \geq 1,\end{cases}
$$

which shows that the $\lim _{n \rightarrow \infty} \frac{\tilde{T}_{n}^{(1)}}{\tilde{c}_{n}}$ does not exist. The scheme considered is realized by the following sequence of random variables $Z_{j}, j \geq 1$ :

$$
Z_{1} \sim \operatorname{Bernoulli}(1 / 2), \quad Z_{j} \sim \operatorname{Po}\left(a_{j}\right), \quad \text { with } a_{j}= \begin{cases}0, & \text { if } j \text { is odd } \\ 2 / j, & \text { if } j \text { is even }\end{cases}
$$

Proposition 4.3 (sufficient condition of convergence/divergence). Let $\tilde{g} \in R T_{\rho}, 0 \leq \rho<\infty$ and let the radius of convergence of the series $\frac{1}{\tilde{g}^{(l)}}$ be greater than $\rho$, for all $l \geq 1$. Then a $R S$ converges if $\left(\tilde{g}^{(l)}(\rho)\right)^{-1}>0, l \geq 1$ and it diverges if $\left(\tilde{g}^{(l)}(\rho)\right)^{-1}=0, l \geq 1$.

Proof. Applying Schur's lemma to (4.3), we get $q^{(l)}=\left(\tilde{g}^{(l)}(\rho)\right)^{-1}<\infty$. By Theorem 3.3 this implies the claim.

Remark 4.4. The example in Remark 4.2 demonstrates the importance of the second condition of Proposition 4.3. In fact, in the model considered $\tilde{g} \in R T_{1}$ and $\left(\tilde{g}^{(1)}(1)\right)^{-1}>0$. However the RS diverges, since the radius of convergence of $\left(\tilde{g}^{(1)}(x)\right)^{-1}=\frac{1}{1+x}$ equals to 1 .

Proposition 4.3 allows to suggest the following two-step strategy for deciding about convergence/divergence of RS's.

(i) Validation of the condition $\tilde{g} \in R T_{\rho}$ for some $0 \leq \rho<\infty$. Our treatment of the problem is based on application of known sufficient conditions on sequences $\left\{m_{j}\right\}_{j \geq 1}$ that guarantee the $R T_{\rho}$ property for the induced sequences $\left\{\tilde{c}_{n}\right\}_{n \geq 0}$. The conditions we employ are the products of two quite different lines of research:

- Burris-Bell theory $[5,6]$ of $R T_{\rho}$ sequences, developed with the help of analytical tools stemming from Tauberian theory. Motivation of this research came from Compton's (1980s) theory of logical limit laws and also from the additive number system theory; 
- Sufficient conditions for $\tilde{g} \in R T_{\rho}$ implied by asymptotic formulae for the number of decomposable structures, in particular recent results by Barbour, Freiman, Stark and the author, derived with the help of probabilistic methods.

Each one of the two approaches has its particular limitations, some of which are noted later on in this Section. In this connection we observe that there is little hope to obtain plausible necessary conditions on $\left\{m_{j}\right\}_{j \geq 1}$ implied by the $R T_{\rho}$ property of $\tilde{g}$.

(ii) Validation of the condition (b) of Schur's lemma for the functions $\left(\tilde{g}^{(l)}\right)^{-1}, l \geq 1$. Provided the condition holds, the conclusion regarding convergence/divergence is based on the claim of

Proposition 4.3.

Following the aforementioned strategy, we examine now the convergence of counting processes for the three basic types of RS's: assemblies, multisets and selections, described in Section 1. Furthermore, the results obtained explain the crucial difference in the asymptotic behaviour of mean-field and non mean-field CFP's associated with the above structures.

First, following [4] we will say that a RS is regularly varying in case it is induced by random variables $Z_{j}, j \geq 1$ in (1.1), with

$$
\mathbb{E} Z_{j} \sim \text { const. } y^{j} j^{\alpha}, \quad \alpha \in \mathbb{R}, \quad y>0, \quad j \rightarrow \infty .
$$

Since the asymptotic behaviour of regularly varying RS's appears to be in accordance with the behaviour of the series $\sum_{j=1}^{\infty} j^{\alpha}$ (see $[3,4,18,22]$ ), it was suggested in [4] to distinguish the following three classes of regularly varying structures: logarithmic $(\alpha=-1)$, convergent $(\alpha<-1)$ and expansive $(\alpha>-1)$. (It goes without saying that in this classification, the meaning of a convergent structure is different from the one in the present paper). As in [18], we extend the above definition of expansive structures to include random structures with $\mathbb{E} Z_{j}, j \geq 1$ oscillating (in $j$ ) between two regularly varying functions, namely

$$
\left(\mathbb{E} Z_{j}, j \geq 1\right) \in \mathcal{F}\left(r_{1}, r_{2} ; y\right):=\left\{f=f(j): \gamma_{1} y^{j} j^{r_{1}-1} \leq f(j) \leq \gamma_{2} y^{j} j^{r_{2}-1}, j \geq 1, y>0\right\},
$$

where $\gamma_{i}, i=1,2$ are positive constants and $0<r_{1} \leq r_{2}$. (The requirement $r_{1}, r_{2}>0$ is the characteristic feature of the expansive case).

Assemblies. Let $Z_{j} \sim \operatorname{Po}\left(a_{j}\right), a_{j}>0, j \geq 1$. In this case,

$$
\tilde{a}_{k_{j}}^{(j)}=\frac{a_{j}^{k_{j}}}{k_{j} !}, \quad \tilde{S}^{(j)}(x)=\exp \left(a_{j} x^{j}\right), \quad j \geq 1
$$

and

$$
\tilde{g}(x)=\exp \left(\sum_{j \geq 1} a_{j} x^{j}\right), \quad \tilde{g}^{(l)}(x)=\exp \left(\sum_{j=1}^{l} a_{j} x^{j}\right), \quad l \geq 1, \quad \mathbb{E} Z_{j}=a_{j}, \quad j \geq 1 .
$$

Thus, the radius of convergence of $\frac{1}{\tilde{g}^{(l)}}$ equals $\infty$ for all finite $l \geq 1$. Consequently, assuming $\tilde{g} \in R T_{\rho}, 0 \leq \rho<\infty$, we have $\left(\tilde{g}^{(l)}(\rho)\right)^{-1}(\rho)>0$. By virtue of Theorem 3.3, the latter leads to the following

Proposition 4.5. An assembly converges if and only if the sequence $\left\{a_{j}\right\}_{j \geq 1}$ is such that $\tilde{g} \in R T_{\rho}$, for some $0 \leq \rho<\infty$.

Corollary 4.6. Assemblies with the following parameter functions $a=\left\{a_{j}, j \geq 1\right\}$ are convergent:

(i) Smoothly growing: $a \in R T_{\rho}, 0<\rho<\infty$;

(ii) Oscillating: $a \in \mathcal{F}\left(\frac{2 r}{3}+\epsilon, r ; y\right)$, where $r, y>0$, while $0<\epsilon \leq \frac{r}{3}$. 
Proof. (i) follows from the important Corollary 4.3 in [6], which says that $a \in R T_{\rho}, 0<\rho<\infty$ implies $\tilde{g} \in R T_{\rho}$, with the same $\rho$. For the proof of (ii), we derive from the asymptotic formula (4.99) in [18] that for the oscillating assembly with $y=1$, we have $\tilde{g} \in R T_{1}$. Hence, it is left to apply (ii) of Remark 3.6 with $\theta=y$.

Note that the restriction on $\epsilon$ in the part (ii) of the last corollary determines a bound on the "size" of oscillation of the function $\mathbb{E} Z_{j}=a_{j}, j \geq 1$ that ensures the $R T_{\rho}$ property for $\tilde{g}$.

Examples. In combinatorics (see Tab. 2.2 in [3]), many assemblies, e.g. permutations $\left(a_{j}=\frac{1}{j}\right)$, Ewens sampling formula $\left(a_{j}=\frac{\theta}{j}, \theta>0\right)$, forests of labeled rooted trees $\left(a_{j}=\frac{j^{j-1}}{j !} \sim\right.$ const. $\left.\mathrm{e}^{j} j^{-\frac{3}{2}}\right)$, etc. are regularly varying, which says that in all these cases $a \in R T_{\frac{1}{y}}, y>0$, where $y$ is as in the definition (4.4) of a regularly varying structure. In statistical mechanics, regularly varying assemblies with $y=1$ and $\alpha>0$ are called generalized Maxwell-Boltzman statistics [31]. By virtue of the condition (i) of Corollary 4.6, all the aforementioned assemblies converge.

Based on Proposition 4.5, we give now two examples of divergent assemblies. Firstly, set partitions $\left(a_{j}=\right.$ $(j !)^{-1}, j \geq 1$ ) diverges, since in this case $\tilde{g}(x)=\mathrm{e}^{e^{x}-1}$, so that the radius of convergence of $\tilde{g}$ is infinity. In this connection note that the following sufficient condition for the $R T_{\infty}$ property of $\tilde{g}$ was recently established in [11]: if the parameter function $a$ is such that $\operatorname{gcd}\left\{j: a_{j}>0\right\}=1$ and $a_{j}=O\left(j^{\theta j} / j !\right), 0<\theta<1, j \rightarrow \infty$, then $\tilde{g} \in R T_{\infty}$, which means that the induced assembly diverges.

For our second example we construct a divergent assembly with $\tilde{g}$ that does not belong to any class $R T_{\rho}, 0 \leq$ $\rho \leq \infty$. It is clear that the corresponding sequence $\left\{a_{j}\right\}_{j \geq 1}$ should exhibit a wild behavior. We set

$$
a_{j}= \begin{cases}j^{-1}, & \text { if } j \geq 1 \text { is odd } \\ j^{-1}+2^{j+1} j^{-1}, & \text { if } j \geq 2 \text { is even. }\end{cases}
$$

We then have

$$
\tilde{g}(x)=\left(\frac{1}{1-x}\right) *\left(\frac{1}{1-4 x^{2}}\right),
$$

which by the Cauchy product formula gives

$$
\tilde{c}_{n}=\sum_{k=0}^{[n / 2]} 4^{k}=\frac{4^{1+[n / 2]}-1}{3}, \quad n \geq 1 .
$$

Consequently,

$$
\begin{gathered}
\lim _{n \rightarrow \infty} \frac{\tilde{c}_{2 n-1}}{\tilde{c}_{2 n}}=1 / 4 \\
\lim _{n \rightarrow \infty} \frac{\tilde{c}_{2 n}}{\tilde{c}_{2 n+1}}=1 .
\end{gathered}
$$

Finally, note that for graphs on $n$ vertices, $\tilde{c}_{n}=\frac{2\left(\begin{array}{c}n \\ 2\end{array}\right)}{n !}$, so that $\tilde{c}_{n} \in R T_{0}$ and by (i) of Remark 3.4, for any $l \geq 1$ the limit measure is concentrated on the singleton $(0, \ldots, 0) \in \mathbb{R}_{l}, l \geq 1$.

We shift now to considering reversible CFP's related to assemblies. In this case the ratio of the net transitions (1.10) has the following form:

$$
q(\eta ; i, j)=V\left(k_{i}, k_{j}, k_{i+j}\right) \begin{cases}\frac{a_{i+j}}{a_{i} a_{j}}, & \text { if } i \neq j: k_{i} k_{j}>0 \\ \frac{a_{2 i}}{a_{i}^{2}}, & \text { if } i=j: k_{i} \geq 2\end{cases}
$$

where

$$
V\left(k_{i}, k_{j}, k_{i+j}\right)= \begin{cases}\frac{k_{i} k_{j}}{k_{i+j}+1}, & \text { if } i \neq j: k_{i} k_{j}>0 \\ \frac{k_{i}\left(k_{i}-1\right)}{k_{2 i}+1}, & \text { if } i=j: k_{i} \geq 2\end{cases}
$$


As we explained in Section 1, such ratios correspond to reversible mean-field CFP's with net transition rates of coagulation and fragmentation as given by (1.11), (1.12) and the equilibrium measure $\mu_{n}$ defined by (1.4) with $Z_{j} \sim P o\left(a_{j}\right), j \geq 1$. Consequently, the preceding discussion reveals that amongst mean-field CFP's both convergent and divergent models exist, depending on the sequence of parameters $\left\{a_{j}\right\}_{1}^{\infty}$.

Multisets. Assuming $Z_{j} \sim N B\left(m_{j}, p^{j}\right), 0<p<1, m_{j} \geq 1, j \geq 1$, we have

$$
a_{k}^{(j)}=\left(1-p^{j}\right)^{m_{j}}\left(\begin{array}{c}
m_{j}+k-1 \\
k
\end{array}\right) p^{j k}, \quad k \geq 0,
$$

where $p$ is a free parameter. This gives

$$
\tilde{a}_{k}^{(j)}=\left(\begin{array}{c}
m_{j}+k-1 \\
k
\end{array}\right) p^{j k}, \quad \tilde{S}^{(j)}(x)=\left(1-(p x)^{j}\right)^{-m_{j}}, \quad|x p|<1,
$$

which leads to the Euler type generating function

$$
\tilde{g}(x)=\prod_{j \geq 1}\left(1-(p x)^{j}\right)^{-m_{j}}, \quad|x|<p^{-1} .
$$

Clearly, the radius of convergence of $\tilde{g}(x)$ is no greater than $p^{-1}$. Moreover, it is known (see e.g. [10], Lem. 1.15) that $\tilde{g}$ converges at some point $x:|x p|<1$ if and only if the sequence $\left\{m_{j}\right\}_{1}^{\infty}$ is such that $\lim _{j \rightarrow \infty}\left(1-(p x)^{j}\right)^{-m_{j}}=1$. Next, in (4.3) the function

$$
\frac{1}{\tilde{g}^{(l)}(x)}=\prod_{j=1}^{l}\left(1-(p x)^{j}\right)^{m_{j}}>0, \quad \text { for all }|x|<p^{-1}, \quad l \geq 1 .
$$

Proposition 4.7. A multiset is convergent if and only if $\tilde{g} \in R T_{\rho}$, for some $\rho<p^{-1}$.

Proof. In the case $\tilde{g} \in R T_{p^{-1}}$, we have $\frac{1}{\tilde{g}^{(l)}\left(p^{-1}\right)}=0, l \geq 1$, which implies divergence. If now $\tilde{g} \in R T_{\rho}$ with $0 \leq \rho<p^{-1}$, then a multiset converges, by Proposition 4.3.

Corollary 4.8. Multisets with the following parameter functions $m=\left\{m_{j}, j \geq 1\right\}$ are divergent:

(i) $m \in R T_{1}$;

(ii) $m_{j}=O\left(j^{\alpha}\right)$, for some $\alpha \in R$;

(iii) $m \in \mathcal{F}\left(\frac{2 r}{3}+\epsilon, r ; 1\right), 0<\epsilon \leq \frac{r}{3}, r>0$, The aforementioned convergence/divergence hold under any $0<p<1$.

whereas multisets with parameter functions (iv)-(vi) below converge:

(iv) $m \in R T_{\rho}$, for some $0<\rho<1$;

(v) $m \in \mathcal{F}\left(\frac{2 r}{3}+\epsilon, r ; y\right), 0<\epsilon \leq \frac{r}{3}, y>1, r>0$;

(vi) $m_{j} \asymp y^{j} j^{-1}, y>1$.

Proof. Each one of the conditions (i) $-(\mathrm{v})$ is sufficient for $\tilde{g} \in R T_{\rho}$ with a corresponding $\rho$. Namely, the conditions (i) and (iv) are due to Bell-Burris Theorems 6.1 and 6.2 of [6], which state that if $m \in R T_{\rho}$, for some $0<\rho \leq 1$, then $\tilde{g}\left(p^{-1} x\right) \in R T_{\rho}$ with the same $\rho$, so that $\tilde{g}(x) \in R T_{\rho p^{-1}}$. (Note that in Thm. 6.1 in [6], condition (c) is required only for the second part of the claim). Condition (ii) provides $\tilde{g}\left(p^{-1} x\right) \in R T_{1}$, by the powerful result of Bell [5] that generalizes the Bateman and Erdös theorem. So, under this condition, $\tilde{g}(x) \in R T_{p^{-1}}$. Conditions (iii) and (v) result from Corollary 2 of [22] for expansive multisets, which says that in both cases $\tilde{g}\left(p^{-1} x\right) \in R T_{y^{-1}}$, which is equivalent to $\tilde{g}(x) \in R T_{y^{-1} p^{-1}}$, with $y \geq 1$. Regarding the condition (vi), we firstly recall that $a_{j} \asymp b_{j}$ means that the ratio $\frac{a_{j}}{b_{j}}, j \geq 1$ is bounded above and below by positive constants. The sufficiency of the condition (vi) for $\tilde{g} \in R T_{y^{-1} p^{-1}}, y>1$ was proven by Stark in [30], which is devoted to logical limit laws for logarithmic multisets. 
Examples. Integer partitions $\left(m_{j}=1, j \geq 1\right)$, planar partitions $\left(m_{j}=j, j \geq 1\right)$ (see [1]) and generalized Bose-Einstein statistics $\left(m_{j}=j^{\alpha}, \alpha>0\right)$ diverge, since in all these cases $m \in R T_{1}$. The following logarithmic multisets (see [3]) with $m \in R T_{\rho}, \rho<1$ converge: mapping patterns $\left(m_{j} \sim \frac{\rho^{-j}}{2 j}, \rho<1, j \geq 1\right.$ ), monic polynomials over $G F(q), q>1\left(m_{j} \sim \frac{q^{j}}{j}\right)$. Also, forests of unlabeled, unrooted $\left(m_{j} \sim\right.$ const. $\left.\rho^{-j} j^{-5 / 2}, \rho<1\right)$ and rooted $\left(m_{j} \sim\right.$ const. $\left.b^{-j} j^{-3 / 2}, b<1\right)$ trees converge.

Remark 4.9. There is a formal linkage between assemblies and multisets, expressed as follows. In the case of assemblies the generating function $\tilde{g}$ is of the exponential form: $\tilde{g}(x)=\mathrm{e}^{Q(x)}$, where $Q(x)=\sum_{j>1} a_{j} x^{j}$, with $a_{j} \geq 0, j \geq 1$. For multisets, the Euler type generating function $\tilde{g}$ in (4.7), can be written in the same form, with the function $Q^{*}$ called the star transformation (see $[5,6]$ ) of the generic generating function $Q(x)=\sum_{j \geq 1} m_{j} p^{j} x^{j}$ of the sequence $\left\{m_{j}\right\}_{j \geq 1}$ :

$$
Q^{*}(x)=\sum_{j \geq 1} m_{j}^{*} x^{j}, \quad m_{j}^{*}=\sum_{l k=j} \frac{m_{l} p^{l}}{k}, \quad j \geq 1, \quad m_{0}^{*}=0 .
$$

Thus, $m_{j}^{*} \geq 0, j \geq 1$, which is a basic assumption in the theory of $R T_{\rho}$ sequences. It was proven in [6] that if $\left\{m_{j} p^{j}\right\}_{j \geq 1} \in R T_{\rho}$, with some $0<\rho<1$, then $m_{j}^{*} \sim m_{j} p^{j}, j \rightarrow \infty$, which means that in this case the multiset behaves asymptotically as the assembly induced by $Z_{j} \sim P o\left(m_{j} p^{j}\right), j \geq 1$. This fact explains the nature of the condition (iv) in Corollary 4.8.

Regarding the CFP's associated with multisets, (1.10) becomes:

$$
q(\eta ; i, j)=V\left(k_{i}, k_{j}, k_{i+j}\right) \begin{cases}\frac{m_{i+j}+k_{i+j}}{\left(m_{i}+k_{i}-1\right)\left(m_{j}+k_{j}-1\right)}, & \text { if } i \neq j: k_{i} k_{j}>0 \\ \frac{m_{2 i}+k_{2 i}}{\left(m_{i}+k_{i}-1\right)\left(m_{i}+k_{i}-2\right)}, & \text { if } i=j: k_{i} \geq 2,\end{cases}
$$

where $V\left(k_{i}, k_{j}, k_{i+j}\right)$ is as in (4.5). The second factor in (4.9) depends both on $\eta=\left(k_{1}, \ldots, k_{n}\right)$ and the parameters $m_{j}, j \geq 1$ of the CFP, so that the representation (1.11) does not hold, which says that the process is not a mean-field model. To illustrate this fact, recall that in a particular case of the $B E$ model $\left(m_{j}=1, j \geq 1\right)$ we saw in Section 1 that the corresponding $\mu_{n}$ is the uniform measure on $\Omega_{n}$, so that we have from (4.9)

$$
q(\eta ; i, j)=1, \eta \in \Omega_{n}, i, j \geq 1, i+j \leq n .
$$

Selections. In this case $Z_{j} \sim B i\left(\frac{p^{j}}{1+p^{j}} ; m_{j}\right), m_{j} \geq O(1), j \rightarrow \infty$ and $0<p \leq 1$. Hence,

$$
\begin{gathered}
\tilde{a}_{k}^{(j)}=\left(\begin{array}{c}
m_{j} \\
k
\end{array}\right) p^{j k}, \\
\tilde{g}(x)=\prod_{j \geq 1}\left(1+(p x)^{j}\right)^{m_{j}} .
\end{gathered}
$$

So, similar to the the case of multisets, $\tilde{g}$ converges at some point $x:|x p|<1$ if and only if the sequence $\left\{m_{j}\right\}_{1}^{\infty}$ is such that $\lim _{j \rightarrow \infty}\left(1+(p x)^{j}\right)^{m_{j}}=1$, which is equivalent to

$$
\lim _{j \rightarrow \infty}(p x)^{j} m_{j}=0 .
$$

Clearly, the radius of convergence, say $\rho$, of $\tilde{g}$ is $\leq p^{-1}$, for any nonnegative sequence $\left\{m_{j}\right\}_{j \geq 1}$. Moreover, the condition implies that $\rho>0$ for all $0<p \leq 1$ if and only if a selection is expansive (see [4,22]), i.e. $m_{j}=O\left(j^{\alpha}\right), \alpha>0$. A majority of practical selections are expansive. 
Proposition 4.10. Selections with radius of convergence $0 \leq \rho<p^{-1}$ converge if and only if the sequence $\left\{m_{j}\right\}_{j \geq 1}$ is such that $\tilde{g} \in R T_{\rho}$. All expansive selections converge.

Proof. In the case of selections, the radius of convergence of the functions

$$
\left(\tilde{g}^{(l)}(x)\right)^{-1}=\prod_{j=1}^{l}\left(1+(p x)^{j}\right)^{-m_{j}}, \quad l \geq 1
$$

is equal to $p^{-1}$, for all $m_{j} \geq 0, j=1, \ldots, l$, while $\frac{1}{\tilde{g}^{(l)}(x)}>0,|x|<p^{-1}$. Consequently, if $\tilde{g} \in R T_{\rho}$ with $\rho<p^{-1}$, then convergence holds by Proposition 4.3. However, if $\rho=p^{-1}$ the second condition of Proposition 4.3 fails, which requires to employ an argument specific for expansive selections. In this latter case $\tilde{c}_{n} \rightarrow \infty$ according to the asymptotic formula derived in [22]. The formula also says that for expansive selections, $\tilde{g} \in R T_{p^{-1}}$. Moreover, following the proof of the formula in [22] it is easy to see that also $\tilde{g}_{\tilde{T}^{(l)}} \in R T_{p^{-1}}$. Writing $\tilde{g}(x)=(1+p x)^{m_{1}} \tilde{g}_{\tilde{T}^{(1)}}$, we get

$$
\tilde{c}_{n}=\sum_{k \geq 0}\left(\begin{array}{c}
m_{1} \\
k
\end{array}\right) p^{k} \tilde{T}_{n-k}^{(1)}, \quad n=1,2, \ldots
$$

With the help of the aforementioned properties of the sequences $\left\{\tilde{c}_{n}\right\}$ and $\left\{\tilde{T}_{n}^{(1)}\right\}$ we are now able to write:

$$
\lim _{n \rightarrow \infty} \frac{\tilde{c}_{n}}{\tilde{T}_{n}^{(1)}}=2^{m_{1}}=\tilde{g}_{\tilde{T}^{(l)}}\left(p^{-1}\right)=\left(q^{(1)}\right)^{-1} .
$$

In an analogous way we get $q^{(l)}=\prod_{j=1}^{l} 2^{-m_{j}}=\left(\tilde{g}_{\tilde{T}^{(l)}}\left(p^{-1}\right)\right)^{-1}, \quad l \geq 1$.

For selections, conditions of Bell-Burris type on $\left\{m_{j}\right\}_{j \geq 1}$, providing $\tilde{g} \in R T_{\rho}$ are not known. This is due to the fact that for selections the star transformation, as defined by (4.8), does not ensure the nonnegativity of $m_{j}^{*}, j \geq 1$. However, the probabilistic method for enumeration of decomposable structures works in the case of selections also. It follows from Theorem 5 in [22], obtained by implementing the method that for expansive selections, $m \in \mathcal{F}\left(\frac{2 r}{3}+\epsilon, r ; y\right), r, y>0$, implies that $\tilde{g} \in R T_{\rho}$, with $\rho=(y p)^{-1}$. By our Proposition 4.7, this provides convergence of the corresponding selections, if $y \geq 1$, under any $0<p<1$. As a result, we derive the convergence of the following expansive selections: integer partitions into distinct parts $\left(m_{j}=1, j \geq 1\right)$ and generalized Fermi statistics $\left(m_{j}=j^{\alpha}, \alpha>0\right)$. In this connection it is in order to note that multisets and selections with $m_{j} \equiv 1$ induce uniform measures $\mu_{n}$ on the set of integer partitions of $n$ and on the set of integer partitions of $n$ into distinct parts, respectively. In the first case the random structure diverges, whereas in the second case convergence to a Bernoulli product measure holds.

For the associated CFP's we obtain from (1.10)

$$
q(\eta ; i, j)=V\left(k_{i}, k_{j}, k_{i+j}\right) \begin{cases}\frac{m_{i+j}-k_{i+j}+1}{\left(m_{i}-k_{i}-1\right)\left(m_{j}-k_{j}-1\right)}, & \text { if } i \neq j: k_{i} k_{j}>0,0 \leq k_{i} \leq m_{i} \\ \frac{m_{2 i}-k_{2 i}}{\left(m_{i}-k_{i}+1\right)\left(m_{i}-k_{i}+2\right)}, & \text { if } i=j: 2 \leq k_{i} \leq m_{i} .\end{cases}
$$

This shows that, as in the case of multisets, the above CFP's are not mean field models.

\section{CONCluding REMARKS AND History}

Generally speaking, the phenomenon of asymptotic independence of a finite number of small groups of particles in large random systems (i.e. systems formed of a large number of randomly interacting particles) was observed in different fields of applications, under various mathematical settings. The assumption of asymptotic independence, sometimes accepted without proof, was of great help for the study of the probabilistic models 
considered. Not pretending to provide a comprehensive survey of the subject, we point out below a few settings parallel (in some sense) to the one in the present paper.

(i) The Gibbs conditioning principle (see $[12,13,15])$. In the context of an ideal gas model, the simplest version of the principle in the title reads as follows. Let $X_{1}, X_{2}, \ldots$ be independent and identically distributed random variables viewed as energies of individual particles, so that $X_{1}+\ldots+X_{n}$ is the total energy of a system of $n$ particles. Let $\mathbb{E} X_{1}=1$ and assume some suitable regularity conditions on a common probability law $P$ of the sequence of random variables. Then, for a fixed $k \geq 1$ the distribution law $\Lambda_{k, n}$ of $\left(X_{1}, \ldots, X_{k}\right)$ conditioned on $X_{1}+\ldots X_{n}=n$ converges weakly, as $n \rightarrow \infty$ to the $k$-fold product law $P^{k}$. In statistical physics the law $\Lambda_{k, n}$ is called a microcanonical distribution, and the gibbs conditional principle asserts asymptotic independence of energies of any finite number of particles in microcanonical ensembles. Formulated in the beginning of the 20th century, the principle has been extended and refined in different directions, with particular attention being paid to the rate of convergence to limit distributions.

To distinguish from the conditioning relation (1.1), the measure $\Lambda_{k, n}$ is defined on the simplex. This fact implies that the Cauchy product relationship (4.2) for generating functions, which is basic for the study of multiplicative measures $\mu_{n}$, is not valid in this case. However, we believe that the interplay between the above two settings deserves further study. Quite independently, the distribution $\Lambda_{k, n}$, with $k=n$ and discrete and not necessary identically distributed random variables $X_{1}, X_{2}, \ldots$ was introduced by Kolchin [26] to represent the distributions of cell counts in combinatorial urn schemes. In [26] the representation is called the generalized scheme of allocation, whereas in $[7,27]$, it is named the Kolchin representation formula. We note that the problem of asymptotic independence is not addressed in [26].

(ii) Random combinatorial structures. In the theory of random structures, the asymptotic independence of counts of small components was discussed in numerous papers, starting from the 1940's. A general set up leading to asymptotic estimation of the total variation distance between component spectra of small counts (as defined by the conditioning relation (1.1)) and the independent process was developed by Arratia and Tavaré in their seminal paper [2] (see also [3,20,26]). As a result, asymptotic independence was established for logarithmic random structures with $y=1$ in (4.4). In [4], the same was proven for regularly varying convergent $(\alpha<-1)$ structures and in [18] the asymptotic independence was proven for expansive assemblies with any $y>0$. Regarding assemblies, multisets and expansive selections, the aforementioned results easily follow from our results in Section 4. In fact, recall that for assemblies $\mathbb{E} Z_{j}=a_{j}, j \geq 1$, for multisets $\mathbb{E} Z_{j}=\frac{m_{j} p^{j}}{1-p^{j}}, j \geq 1,0<p<1$ and for selections $\mathbb{E} Z_{j}=\frac{m_{j} p^{j}}{1+p^{j}}, j \geq 1,0<p<1$. By the definition (4.4) of a regularly varying RS with parameters $y>0, \alpha \in \mathbb{R}$ we thereby conclude that the following facts hold.

- For assemblies: $a=\left\{a_{j}, j \geq 1\right\} \in R T_{y^{-1}}, y>0$, for all $\alpha \in \mathbb{R}$, which implies convergence by condition (i) of Corollary 4.6;

- for multisets: $m=\left\{m_{j}, j \geq 1\right\} \in R T_{y^{-1} p}, 0<p<1$, for all $\alpha \in R$. This implies convergence if $y \geq 1$, by the condition (iv) of Corollary 4.8. Note that in the case $y=p$, a regularly varying multiset diverges for all $\alpha \in \mathbb{R}$, by condition (i) of Corollary 4.8 ;

- for selections: $m_{j} \sim$ const. $j^{\alpha}\left(y p^{-1}\right)^{j}, j \geq 1$. By the discussion following Proposition 4.7, this provides convergence for all $\alpha>0$ and $y \geq 1$.

In this connection, we mention that to our knowledge no examples of RS's for which the independence principles fails were given in the literature, prior to this paper.

Our results reveal also a basic difference between pictures of asymptotic clustering of components in convergent RS's and in regularly varying divergent multisets. Namely, in the case of a convergent RS with $\rho \neq 0$, Corollary 3.5 tells us that with a positive limit probability there are components of any fixed sizes, i.e.

$$
\lim _{n \rightarrow \infty} \mathbb{P}\left(K_{1}^{(n)}=k_{1}, \ldots, K_{l}^{(n)}=k_{l}\right)>0, \quad k_{1}, \ldots, k_{l} \geq 0, \quad l \geq 1 .
$$

On the other hand, if a regularly varying multiset diverges, then $q^{(l)}=0, l \geq 1$, by Proposition 4.5, from which it follows that $\lim _{n \rightarrow \infty} \mathbb{P}\left(K_{j}^{(n)}=k_{j}\right)=0$, for all finite $k_{j} \geq 0, j \geq 1$. 
(iii) CFP's. It is common to trace the beginning of rigorous mathematical models of coagulation-fragmentation back to the paper by Smoluchowski (1918) where the famous system of coagulation equations describing the time evolution of the process was derived. Already in this paper the assumption of independence (more precisely, the absence of correlations) of clusters of small sizes was adopted. Subsequently, deterministic and stochastic versions of the model were studied in numerous papers in probability and various applied fields. The study of reversible CFP's was concentrated on what we call in the present paper mean-field CFP's (see [25,33]). (Recall that these models conform to assemblies). In [14] the model was treated as a reversible Markov chain on the set of partitions and it was proven (Thm. 4, (4.24)) that, if $\tilde{g} \in R T_{\rho}, 0 \leq \rho<\infty$, then at the equilibrium of the process,

$$
\operatorname{cov}\left(K_{l}^{(n)}, K_{m}^{(n)}\right) \rightarrow 0, n \rightarrow \infty
$$

for any fixed $l \neq m$. This is, of course, a weak form of our Proposition 4.5. More details on the history of CFP's can be found in $[18,19]$.

\section{(iv) Convergence of scaled counting processes.}

In the setting of the present paper, as well as in the all above mentioned settings, the convergence of generic (=nonscaled) counting processes was studied. Our Theorem 3.3 asserts that a generic spectrum either converges (in distribution) to a distribution with independent components, or diverges. If the first option is the case, then the simple discrete limiting process provides approximation of the discrete generic one. In some (but not all!) cases of RS's it is possible to find a proper scaling that secures convergence. The disadvantage of approximation in this latter case is that the limiting process is no longer lies in $\mathcal{N}$. Some examples of scaling of logarithmic RS's are presented e.g. in [3]. A key role here is played by Poisson-Dirichlet distribution on a simplex as a limit of a scaled Ewens sampling formula. In [17], it was proven a general result from which follows (Cor. 3.1 there) that nondegenerate limiting distributions are possible for convergent and logarithmic RS's only. In the case of expansive RS's the limiting distribution of a properly scaled counting process is known to be a curve called a limit shape of a random Young diagram (for the history of limit shapes see the recent papers [17,34]. Yakubovich [34] established general conditions for the existence of limit shapes for scaled multiplicative measures that encompass known results on limit shapes for regularly varying RS's.

Acknowledgements. This research was supported by New York Metropolitan Research Fund. A part of the present paper was written during my visit to the School of Mathematical Sciences, at Monash University. I am grateful to Fima Klebaner, Aiden Sudbury and Kais Hamza for their hospitality. I would also like to express my gratitude to a referee for important critical remarks and suggestions.

\section{REFERENCES}

[1] G. Andrews, The theory of partitions, Encyclopedia of Mathematics and its Applications. Addison-Wesley 2 (1976).

[2] R. Arratia and S. Tavaré, Independent process approximations for random combinatorial structures. Adv. Math. 104 (1994) 90-154.

[3] R. Arratia, A. Barbour and S. Tavaré, Logarithmic combinatorial structures: a probabilistic approach. European Mathematical Society Publishing House, Zurich (2004).

[4] A. Barbour and B. Granovsky, Random combinatorial structures: the convergent case. J. Comb. Theory, Ser. A 109 (2005) $203-220$.

[5] J. Bell, Sufficient conditions for zero-one laws. Trans. Amer. Math. Soc. 354 (2002) 613-630.

[6] J. Bell and S. Burris, Asymptotics for logical limit laws: when the growth of the components is in RT class. Trans. Amer. Soc. 355 (2003) 3777-3794.

[7] N. Berestycki and J. Pitman, Gibbs distributions for random partitions generated by a fragmentation process. J. Stat. Phys. 127 (2006) 381-418.

[8] J. Bertoin, Random fragmentation and coagulation processes, Cambridge Studies in Advanced Mathematics. Cambridge University Press (2006).

[9] B. Bollobás, Random graphs, Cambridge Studies in Advanced Mathematics. Cambridge University Press (2001).

[10] S. Burris, Number theoretic density and logical limit laws, Mathematical Surveys and Monographs. American Mathematical Society, Providence, RI 86 (2001).

[11] S. Burris and K. Yeats, Sufficient conditions for labelled 0-1 laws. Discrete Math. Theory Comput. Sci. 10 (2008) 147-156. 
[12] P. Cattiaux and N. Gozlan, Deviations bounds and conditional principles for thin sets. Stoch. Proc. Appl. 117 (2007) 221-250.

[13] A. Dembo and O. Zeitouni, Refinements of the Gibbs conditioning principle. Probab. Theory Relat. Fields 104 (1996) 1-14.

[14] R. Durrett, B. Granovsky and S. Gueron, The equilibrium behaviour of reversible coagulation-fragmentation processes. J. Theoret. Probab. 12 (1999) 447-474.

[15] P. Diaconis and D. Freedman, Conditional limit theorems for exponential families and finite versions of de Finetti's theorem. J. Theoret. Probab. 1 (1988) 381-410.

[16] M. Erlihson and B. Granovsky, Reversible coagulation-fragmentation processes and random combinatorial structures: asymptotics for the number of groups. Random Struct. Algorithms 25 (2004) 227-245.

[17] M. Erlihson and B. Granovsky, Limit shapes of multiplicative measures associated with coagulation-fragmentation processes and random combinatorial structures. Ann. Inst. Henri Poincaré Prob. Stat. 44 (2005) 915-945.

[18] G. Freiman and B. Granovsky, Asymptotic formula for a partition function of reversible coagulation-fragmentation processes. J. Israel Math. 130 (2002) 259-279.

[19] G. Freiman and B. Granovsky, Clustering in coagulation-fragmentation processes, random combinatorial structures and additive number systems: asymptotic formulae and limiting laws. Trans. Amer. Math. Soc. 357 (2005) 2483-2507.

[20] B. Fristedt, The structure of random partitions of large integers. Trans. Amer. Math. Soc. 337 (1993) $703-735$.

[21] B. Granovsky and A. Kryvoshaev, Coagulation processes with Gibbsian time evolution. arXiv:1008.1027 (2010).

[22] B. Granovsky and D. Stark, Asymptotic enumeration and logical limit laws for expansive multisets. J. London Math. Soc. 73 (2005) 252-272.

[23] W. Greiner, L. Neise and H. Stöcker, Thermodinamics and Statistical Mechanics, Classical Theoretical Physics. Springer-Verlag (2000).

[24] E. Grosswald, Representatin of integers as sums of squares. Springer-Verlag (1985).

[25] F. Kelly, Reversibility and stochastic networks. Wiley (1979).

[26] V. Kolchin, Random graphs, Encyclopedia of Mathematics and its Applications. Cambridge University Press 53 (1999).

[27] J. Pitman, Combinatorial stochastic processes. Lect. Notes Math. 1875 (2006).

[28] G. Polya and G. szego, Problems and Theorems in Analysis, Vol. VI. Springer-Verlag (1970).

[29] L. Salasnich, Ideal quantum gas in D-dimensional space and power law potentials, J. Math. Phys. 41 (2000) $8016-8024$.

[30] D. Stark, Logical limit laws for logarithmic structures, Math. Proc. Cambridge Philos. Soc. 140 (2005) 537-544.

[31] A. Vershik, Statistical mechanics of combinatorial partitions and their limit configurations. Funct. Anal. Appl. 30 (1996) 90-105.

[32] A. Vershik and Yu. Yakubovich, Fluctuations of the maximal particle energy of the quantum ideal gas and random partitions. Commun. Math. Phys. 261 (2006) 759-769.

[33] P. Whittle, Systems in stochastic equilibrium. Wiley (1986).

[34] Yu. Yakubovich, Ergodicity of multiple statistics. arXiv:0901.4655v2 [math.C0] (2009).

[35] K. Yeats, A multiplicative analogue of Schur's Tauberian theorem. Can. Math. Bull. 46 (2003) 473-480. 